\title{
Ensaio de Usabilidade de uma Intervenção Psicoeducacional Computadorizada sobre Transtorno Obsessivo-Compulsivo
}

\author{
Gerson Siegmund ${ }^{1}$ \\ Roberto Guedes de Nonohay \\ Programa de Pós-Graduação em Psicologia da Universidade Federal do Rio Grande do Sul, \\ Porto Alegre, RS, Brasil \\ Gustavo Gauer \\ Departamento de Psicologia do Desenvolvimento e da Personalidade da Universidade \\ Federal do Rio Grande do Sul, Porto Alegre, RS, Brasil
}

\section{Resumo}

O objetivo deste trabalho foi avaliar a usabilidade, funcionalidade e efeitos iniciais de uma intervenção psicoeducacional computadorizada sobre Transtorno Obsessivo-Compulsivo (TOC), através de um ensaio com usuários. A intervenção consistiu em um programa composto por três módulos contendo textos informativos, vídeos e três quizzes com 10 perguntas cada. Participaram 21 sujeitos entre 19 e 55 anos. Os instrumentos utilizados foram o Yale-Brown Obsessive-Compulsive Scale (Y-BOCS), escalas subjetivas para avaliação da intensidade dos sintomas de TOC, escalas subjetivas de humor e de ansiedade, questões de usabilidade e log do programa. Os participantes levaram em média oito dias para completar o programa e o tempo médio de uso totalizou 2 horas e 14 minutos. Apenas uma questão dos quizzes teve frequência de acertos abaixo de 70\%. O nível médio de satisfação foi de 8,33 no primeiro módulo, 8,71 no segundo e 9,00 no terceiro. Houve diminuição nos escores obsessivos da Y-BOCS e diferença estatisticamente significativa na escala subjetiva de sintomas do TOC entre os módulos 1 e 2, e 1 e 3. O programa obteve um bom nível de satisfação dos usuários e apresenta potencial efeito na redução de sintomas percebidos. Entende-se que o desenvolvimento do programa contribui em termos teóricos e práticos para o atual momento da psicologia e sua interface com a tecnologia.

Palavras-chave: Transtorno Obsessivo-Compulsivo, psicoeducação, psicologia online, terapia cognitivo-comportamental, intervenções computadorizadas.

\section{Usability Trial of a Computerized Psychoeducational Intervention about Obsessive-Compulsive Disorder}

\footnotetext{
Abstract

This study aimed at evaluating usability, functionality and initial effects of a computerized psychoeducational intervention directed at Obsessive-Compulsive Disorder (OCD), through a usability

Endereço para correspondência: Universidade Federal do Rio Grande do Sul, Instituto de Psicologia, sala 123, R. Ramiro Barcelos, 2600, Bairro Santana, Porto Alegre, RS, Brasil 90035-003. Fone: (51) 3308-5303 / (51) 8247-6627, Fax: (51) 3022-1513. E-mail: siegmund.gerson@gmail.com, nonohay@hotmail.com e gusgau@ gmail.com

Este trabalho recebeu apoio da Coordenação de Aperfeiçoamento de Pessoal de Nível Superior (CAPES) através de bolsa de Mestrado concedida ao primeiro autor.
} 
trial with users. Twenty-one users ranging from 19 to 55 years old took part in the study. Measures were Yale-Brown Obsessive-Compulsive Scale (Y-BOCS), subjective scales to assess OCD symptoms intensity, mood and anxiety, usability questions, and the system log. Participants took an average of eight days to complete the intervention, and the average time of software usage was 2 hours and 14 minutes. Only one quiz question showed less than $70 \%$ correct answers. Mean level of satisfaction was 8.33 for the first module, 8.71 for the second and 9.00 for the third. There was a decrease in Y-BOCS obsessive scores, and a statistically significant difference was found on the scale of OCD symptoms intensity, between modules 1 and 2, and 1 and 3. The software reached a good level of satisfaction among users and shows potential in reduction of perceived symptoms. It is argued that, as a template, the program can contribute both practically and theoretically for novel applications in the interface of psychotherapy and technology.

Keywords: Obsessive-Compulsive Disorder, psychoeducation, telepsychology, Cognitive-Behavioral Therapy, computerized interventions.

\section{Test de Usabilidad de una Intervención Psicoeducativa Computadorizada sobre el Transtorno Obsesivo-Compulsivo}

\section{Resumen}

El objetivo de este estudio fue evaluar la usabilidad, funcionalidad y efectos iniciales de una intervención psicoeducativa computarizada sobre el Trastorno obsesivo-compulsivo (TOC), a través de un experimento con usuarios. Participaron 21 sujetos con edades entre 19 y 55 años. Los instrumentos utilizados fueron lo Yale-Brown Obsessive-Compulsive Scale (Y-BOCS), escalas subjetivas para evaluar la gravedad de los síntomas del TOC, el estado de ánimo y la ansiedad, preguntas de usabilidad y el registro del programa. Los participantes tomaron un promedio de ocho días para completar el programa, y el tiempo de uso promedio fue de dos horas y 14 minutos. Sólo una cuestión de los quizzes tuvo un promedio de respuestas correctas abajo del $70 \%$. El nivel medio de satisfacción fue 8.33 en el primer módulo, 8.71 en el segundo y 9.00 en el tercero. Hubo una reducción en la subescala de síntomas obsesivos del Y-BOCS y una diferencia estadísticamente significativa en la escala subjetiva de los síntomas del TOC entre los módulos 1 y 2, y 1 y 3 . El programa logró un buen nivel de satisfacción entre los usuarios y presenta potencial para la reducción de los síntomas percibidos. Se entiende que el desarrollo del programa contribuye en términos teóricos y prácticos para el momento actual de la psicología y su interfaz con la tecnología.

Palabras clave: Trastorno Obsesivo-Compulsivo, psicoeducación, psicología online, terapia cognitivo-conductual, intervención computarizada.

A interface entre psicologia e a tecnologia tem como um de seus focos o uso da Internet e dos avanços tecnológicos como ferramentas para o oferecimento de intervenções psicológicas (Castelnuovo, Gaggioli, Mantovani, \& Riva, 2003). No Brasil, a resolução 011/2012 regulamenta as práticas e serviços psicológicos mediados por computador (Conselho Federal de Psicologia [CFP], 2012), estabelecendo como prática legal e passível de remuneração a orientação psicológica online. Esta prática equivale- ria a um aconselhamento pontual, informativo e focado no problema proposto, ocorrendo em um limite máximo de vinte sessões. Por outro lado, a mesma legislação proíbe ao profissional de psicologia realizar qualquer forma de terapia pela Internet, exceto em caráter declaradamente experimental. O posicionamento do CFP reflete a carência de estudos sobre o tema no Brasil. Não obstante, um número crescente de estudos sobre terapia pela Internet vem sendo desenvolvido em outros países e seus resultados podem 
ser utilizados como referência para o desenvolvimento de tratamentos computadorizados no Brasil.

A terapia cognitivo-comportamental (TCC) oferecida via Internet tem apresentado indícios de efetividade para uma variedade de problemas psicológicos e comportamentais, como depressão (Titov, Andrews, \& Sachdev, 2010; Vernmark et al., 2010) e transtornos de ansiedade (Klein et al., 2010; Pier et al., 2008). A quantidade e a qualidade dos estudos sugerem que a prática da TCC pela Internet continuará acontecendo e é plausível considerar que a Internet será utilizada, de alguma forma, em todos os tratamentos futuros (G. Andersson, 2009). Em especial, o tratamento para Transtorno Obsessivo-Compulsivo (TOC) pode contar com o auxílio da tecnologia para potencializar seus benefícios.

\section{Tratamento e Acessibilidade}

O TOC é uma doença relativamente comum, que atinge cerca de $2 \%$ a $3 \%$ das pessoas ao longo da vida (Crino, Slade, \& Andrews, 2005). $\mathrm{O}$ transtorno se manifesta através de obsessões e compulsões e causa significativo prejuízo na vida do indivíduo, consumindo mais de uma hora por dia do tempo do indivíduo e gerando acentuado sofrimento (American Psychiatric Association [APA], 2013).

A terapia comportamental, de exposição e prevenção de resposta (EPR) e sua associação com terapia cognitiva vem demonstrando ótimos resultados (Foa et al., 2005; Franklin, Abramowitz, Bux, Zoellner, \& Feeny, 2002), mas uma pesquisa realizada nos Estados Unidos mostra que, em geral, existe um baixo índice de acesso ao tratamento por parte da população (Goodwin, Koenen, Hellman, Guardino, \& Struening, 2002). Alguns dos principais motivos são a falta de diagnóstico apropriado, a escassez de profissionais qualificados, a falta de terapeutas cognitivo-comportamentais nos sistemas públicos de saúde e receios do paciente quanto ao tratamento (Goodwin et al., 2002; Herbst et al., 2012; Shapiro, Cavanagh, \& Lomas, 2003). O atendimento via Internet se apresenta como um meio efetivo de superar estes obstáculos.

\section{Terapia Cognitivo-Comportamental Online para TOC}

Intervenções pela Internet apresentam diversas vantagens, tais como a opção do anonimato, a economia de tempo dos profissionais e principalmente a possibilidade de atendimento à distância. Os ensaios sobre TCC online para TOC demonstram melhora significativa dos sintomas, além de melhora no funcionamento geral e aceitação ao tratamento online (E. Andersson et al., 2012; E. Andersson et al., 2011; Wootton et al., 2011). No entanto, grande parte dos estudos foram realizados com o acompanhamento direto de um terapeuta.

Considerados os dados iniciais, autores da área sugerem que é valido o esforço de investir em pesquisas sobre tratamentos computadorizados autoadministrados para TOC (Barlow, Ellard, Hainsworth, Jones, \& Fisher, 2005). Outra revisão de literatura abrangente sobre os tratamentos online do transtorno obsessivo-compulsivo mostrou que poucas pesquisas foram realizadas, tendo sido encontrados 22 estudos, dos quais apenas três consistiram no uso de programas de computador automatizados (Herbst et al., 2012). Em sua maioria, os resultados dos estudos foram favoráveis, apresentando redução dos sintomas de TOC. No entanto, há diversidade nas metodologias utilizadas, não sendo possível formar conclusões decisivas. Nenhum desses estudos teve como foco a psicoeducação.

\section{Psicoeducação}

A psicoeducação, entendida como o processo de educar o paciente sobre seu transtorno ou funcionamento é um dos princípios básicos da terapia cognitiva (Beck, 2007), recebendo destaque especial no tratamento de pessoas com esquizofrenia (Lehman et al., 2004), transtorno bipolar (Colom, 2014) e depressão (Dowrick et al., 2000). Intervenções psicoeducacionais para transtornos envolvendo ansiedade são geralmente bem aceitas e avaliadas como úteis pelos pacientes (Houghton \& Saxon, 2007). Em se tratando do TOC, a psicoeducação tem o objetivo amplo de melhorar a assimilação do paciente à lógica do tratamento. Neste caso, o 
componente educacional pode não apenas melhorar a adesão aos tratamentos psicológico e farmacológico, mas também atuar na correção de crenças errôneas sobre o medo e ansiedade (Rachman, 2006).

A APA estimula a psicoeducação no TOC, objetivando que a doença seja desestigmatizada e o paciente possa tomar decisões com mais informações sobre a situação (Koran et al., 2007). Resultados de um estudo sobre EPR para TOC indicaram que os pacientes que entenderam melhor a lógica do tratamento seguiram melhor as instruções de exposição, levando à maior redução de sintomas (Abramowitz, Franklin, Zoellner, \& DiBernardo, 2002). Novamente, uma revisão de literatura recente sugeriu que as novas tecnologias de informação podem ser aproveitadas para oferecer psicoeducação computadorizada sobre TOC (Lind, Boschen, \& Morrissey, 2013). No entanto, para que um programa de Psicologia seja efetivo, ele deve atender aos critérios da usabilidade que são comuns a todo software e independentes do seu conteúdo.

\section{Avaliação de Usabilidade}

O conceito de usabilidade é oriundo da ciência da computação e ela pode ser definida como a capacidade que um sistema tem de permitir que o usuário consiga alcançar objetivos específicos com segurança, efetividade, eficiência e satisfação (International Organization for Standardization [ISO] 9241-11, 1998; Preece et al., 1994). Para alcançar um alto nível de usabilidade, o programa deve ser (a) eficaz: alcançar o resultado esperado com acurácia e completude; (b) eficiente: o resultado deve ser alcançado de forma rápida e abrangente, com o mínimo de tempo e custos possível; (c) satisfatório: produzir uma atitude de aceitação e minimizar o desconforto (ISO 9241-11, 1998).

Em estudos que buscam criar um novo soft- ware ou sistema, os usuários potenciais participam do processo de desenvolvimento em um ou mais momentos. Delineamentos semelhantes vem sendo utilizados no desenvolvimento de intervenções de saúde pela web, seja na saúde em geral (Corrao, Robinson, Swiernik, \& Naeim, 2010; Kim \& Kang, 2013; Patterson et al., 2013; Rhebergen, Hulshof, Lenderink, \& van Dijk, 2010; Riiser, Løndal, Ommundsen, Sundar, \& Helseth, 2013) ou na psicologia (Anderson, Zimand, Schmertz, \& Ferrer, 2007; Currie, McGrath, \& Day, 2010). Quando se trata de intervenções psicológicas é fundamental utilizar esse tipo de avaliação para que o sistema fique agradável e atraente para os futuros usuários (Currie et al., 2010).

\section{Objetivos}

O objetivo deste estudo foi realizar um ensaio com usuários do público em geral para avaliar os principais aspectos de usabilidade de uma intervenção psicoeducacional computadorizada sobre TOC. Esperava-se que os usuários fornecessem avaliações úteis à reformulação do programa, no que toca ao seu conteúdo e também à sua estrutura e funcionalidade. Também buscou-se avaliar os efeitos iniciais da intervenção enquanto redução de sintomas obsessivos-compulsivos.

\section{Metodologia}

\section{Delineamento}

Trata-se de um estudo de caráter experimental no qual foi realizado um ensaio aberto de uma intervenção computadorizada, com foco na usabilidade e avaliações em diferentes momentos. Este estudo faz parte de um projeto maior de desenvolvimento de software, que seguiu a estrutura descrita na Figura 1. Dado que o objetivo deste manuscrito foi avaliar a usabilidade e os efeitos iniciais da intervenção, optou-se por apresentar apenas a terceira fase do desenvolvimento.

\section{Participantes}

Participaram do estudo 21 pessoas da população em geral, homens e mulheres. Uma vez que o foco deste estudo foi a avaliação da usabilidade e considerando que o programa pode ser utilizado tanto por pessoas com TOC quanto usuários saudáveis, definiu-se não ser imprescindível que os participantes apresentassem sintomas de TOC. Os critérios de inclusão foram: (a) ter entre 18 e 60 anos; (b) saber utilizar um 


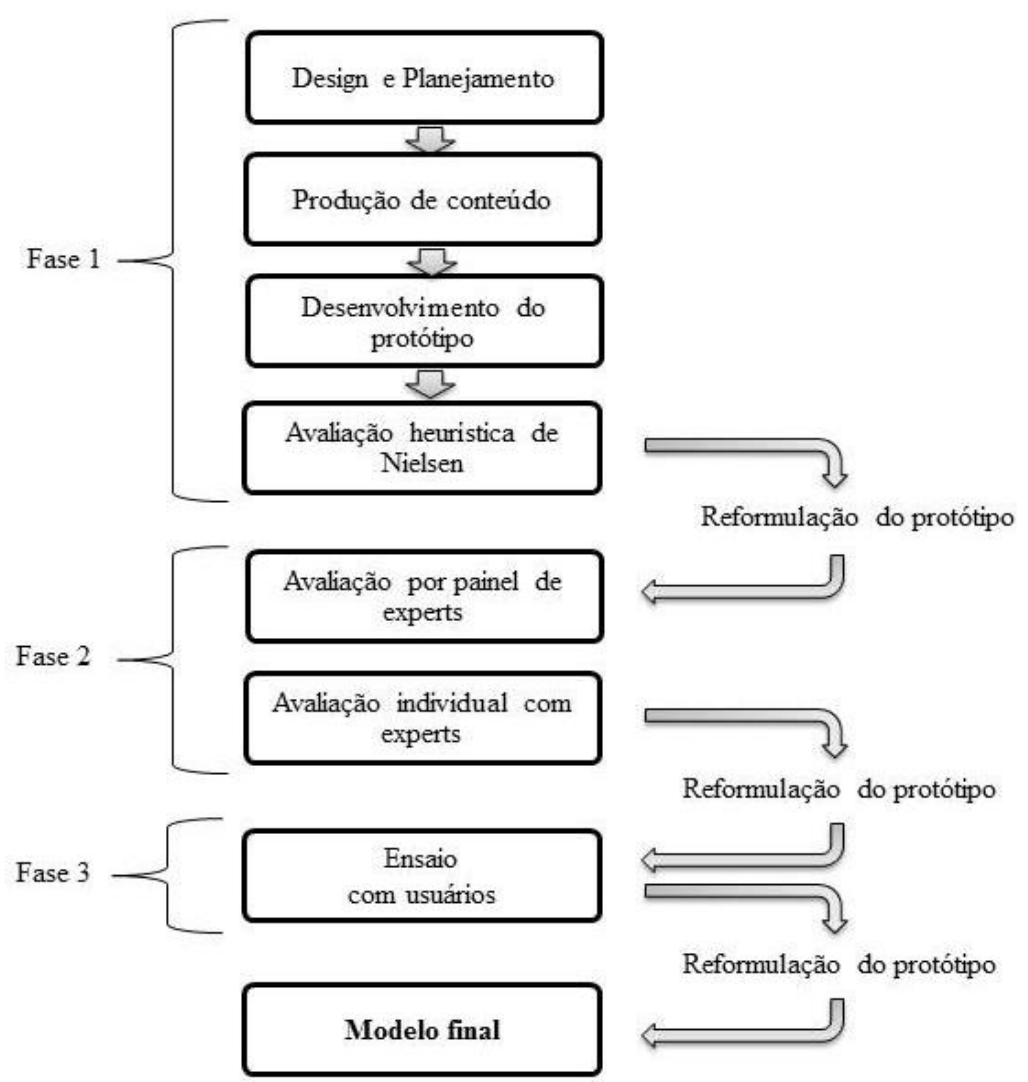

Figura 1. Sequência das fases de desenvolvimento do programa.

computador; (c) ter acesso a um computador com Internet para receber e enviar os arquivos; e (d) ter o Microsoft Excel instalado em seu computador.

\section{Procedimentos}

A divulgação da pesquisa foi feita através de redes sociais e listas de e-mail. Das 45 pessoas que se voluntariaram, cinco não preencheram os critérios de inclusão e 19 não enviaram o programa. Os participantes interessados assinaram o Termo de Compromisso, além de responderem presencialmente a um questionário sociodemográfico e um questionário de habilidade com tecnologia. Os participantes receberam o programa por e-mail e um arquivo contendo instruções para utilização. O telefone do pesquisador foi disponibilizado para dúvidas e auxílio técnico. Após o envio do programa, foi realizado um monitoramento por e-mails a cada sete, 14 e 17 dias (sendo 17 dias o prazo final para o usuário não ser excluído da pesquisa). Nestes e-mails o pesquisador questionava o usuário a respeito da utilização do programa e se colocava novamente à disposição para esclarecer dúvidas ou questões técnicas.

\section{Instrumentos e Materiais}

Yale-Brown Obsessive Compulsive Scale (Y-BOCS; Goodman et al., 1989). Instrumento frequentemente utilizado, é considerado padrão-ouro para avaliação da gravidade dos sintomas de TOC. Foi traduzida para o português por Asbahr et al. (1992). Conta com 10 questões sobre obsessões e compulsões, cujas respostas variam de 0 (sem sintomas) a 4 (muito grave). A aplicação ocorreu ao final do primeiro e ao final do último módulo. Optou-se por realizar a aplicação ao final do primeiro módulo para que os usuários respondessem ao instrumento com mais conhecimento sobre o tema..

Avaliação Subjetiva de Sintomas do TOC. O usuário era solicitado a dar uma nota representando a intensidade dos seus sintomas de TOC naquele dia. A escala variava de 0 ("sem sintomas") a 7 ("muito intenso"). O usuário 
podia selecionar a nota correspondente clicando na caixa que se abria, ou digitando diretamente o número no teclado. A mesma estrutura foi aplicada na avaliação do humor e da ansiedade. Esta avaliação aconteceu no início de cada módulo, também juntamente com a de humor e de ansiedade.

Avaliação Subjetiva de Humor. O usuário era solicitado a dar uma nota representando seu humor naquele dia. A escala variava de 0 ("sentindo-se muito triste, sem energia, sem vontade de fazer as coisas") a 10 ("sentindo-se muito feliz, com disposição e vontade de fazer muitas atividades").

Avaliação Subjetiva de Ansiedade. O usuário era solicitado a dar uma nota representando sua ansiedade naquele dia. A escala variava de 0 ("nenhuma ansiedade, totalmente tranquilo, despreocupado") a 10 ("muita ansiedade, totalmente agitado, preocupado, ou nervoso").

Questões de Usabilidade. Foram utilizadas cinco questões de usabilidade, sobre efetividade, eficiência, compreensão textual, confortabilidade e satisfação. Exemplo de questão: "Os textos deste módulo estão compreensíveis?". Para essas questões, o usuário poderia responder "sim" ou "não". Uma última questão avaliava o grau de satisfação com o módulo, de 1 a 10. Essa avaliação aconteceu ao final de cada módulo.

Log. O sistema armazenou registros da utilização, tais como tempo de uso em cada módulo, tempo de uso geral, intervalo entre acessos, frequência, realização de tarefas, acertos no quiz, respostas, etc.

Microsoft Excel. A plataforma utilizada para rodar o programa foi o Microsoft Excel, nas versões 97 a 2007, através da linguagem VBA (Visual Basic Applications).

\section{Descrição da Intervenção}

A intervenção consistiu na utilização do ProTOC, um programa de tratamento cognitivocomportamental computadorizado para pessoas com sintomas de Transtorno ObsessivoCompulsivo. O programa original é composto por 12 módulos, que incluem psicoeducação, exposição e prevenção de respostas e reestru- turação cognitiva. Para este estudo foi utilizada apenas a seção de psicoeducação, composta por três módulos. Os módulos foram sequenciais, onde o usuário só poderia avançar para o módulo seguinte quando completasse $\mathrm{o}$ anterior. Não houve acompanhamento de terapeuta. $\mathrm{O}$ conteúdo foi textual, procurou-se que tivesse feedbacks automáticos, não dependesse de acompanhamento terapêutico e pudesse ser utilizado no ritmo do usuário.

Nas primeiras páginas, o usuário tinha a possibilidade de inserir o nome pelo qual gostaria de ser chamado. A partir de então, eventualmente o programa se dirigia ao usuário chamando-o por este nome. No início de cada módulo o usuário era solicitado a avaliar seu humor, ansiedade e sintomas percebidos de TOC através de uma nota, a partir da qual um feedback na janela indicava a interpretação dessa pontuação. Por exemplo, ao selecionar sintomas de TOC em nível 7 a mensagem era "Ok, seus sintomas estão em um nível alto". Eventualmente o programa reforçava o usuário através de mensagens no texto, como por exemplo: "Ótimo! Que bom que você chegou até aqui”. Todas as páginas continham um botão "Prosseguir" que levava à página seguinte e um botão "Sair" que encerrava o programa. Caso optasse por sair, uma mensagem automática surgia na tela dizendo que não havia problema, mas que em caso de dúvidas ou intenção de não participar da pesquisa, fosse avisado ao pesquisador através do contato disponibilizado.

Em todas as páginas o tempo de uso era livre, de forma que o programa só avançava à medida que se clicasse em "Prosseguir". O programa estimulava a utilização dentro do prazo de uma semana, o que era expresso ao final de cada módulo com uma mensagem de incentivo à utilização em poucos dias. Inicialmente, o tempo mínimo de utilização era de três dias, pois uma trava de 24 horas entre cada módulo limitava a utilização do programa. Foram abordados os seguintes tópicos:

1. Módulo 1 (elementos básicos): o que é o TOC, o que são obsessões e compulsões, qual a diferença entre TOC e manias consideradas normais. 
2. Módulo 2 (aprofundamento): base neurobiológica do TOC, origem e manutenção dos medos, relação entre obsessões e compulsões, explicação dos modelos cognitivo e comportamental para o TOC, o que são crenças e distorções cognitivas.

3. Módulo 3 (TCC): o que é a terapia cognitivo-comportamental, interligação entre pensamentos, emoções e comportamentos, características principais da TCC e como funciona o tratamento nessa modalidade.

Ao final de cada módulo, foi aplicado um quiz contendo 10 perguntas de múltipla escolha sobre os assuntos recém trabalhados, contendo uma alternativa correta e três incorretas. As respostas no quiz geravam um feedback automático, no formato de uma caixa de diálogo do windows. Quando a resposta era correta, o feedback informava que a resposta estava certa. Quando a resposta era incorreta, o feedback informava que a resposta estava errada e indicava a resposta correta. Durante o quiz não era possível retornar para responder uma questão separadamente. Ao final, o usuário era informado do seu número de acertos. Se acertasse menos de seis questões, podia optar entre refazer o quiz naquele momento ou rever o conteúdo. Se acertasse seis ou mais podia optar por refazer o quiz ou continuar para a próxima janela.

Cada módulo continha um sumário do conteúdo, uma revisão de humor, ansiedade e sintomas de TOC, o quiz e seu respectivo resultado, um resumo do módulo e a indicação de uma tarefa extra. As tarefas foram três vídeos: o primeiro mostrava a entrevista de um artista famoso falando sobre seus sintomas de TOC e os seguintes ilustravam a rotina de alguém com sintomas de TOC, especialmente compulsões de perfeccionismo e checagem. O programa oferecia um link direto para o vídeo no site youtube. No último módulo, uma indicação era realizada com base na pontuação da Y-BOCS: em caso de sintomas leves (até 15 pontos no escore total), era sugerido que a pessoa realizasse uma avaliação com psicólogo ou psiquiatra. Em caso de sintomas graves (acima de 15 pontos), a sugestão era de procurar um especialista imediatamente. A Figura 2 mostra uma pergunta do primeiro quiz, para ilustrar o aspecto visual do programa.
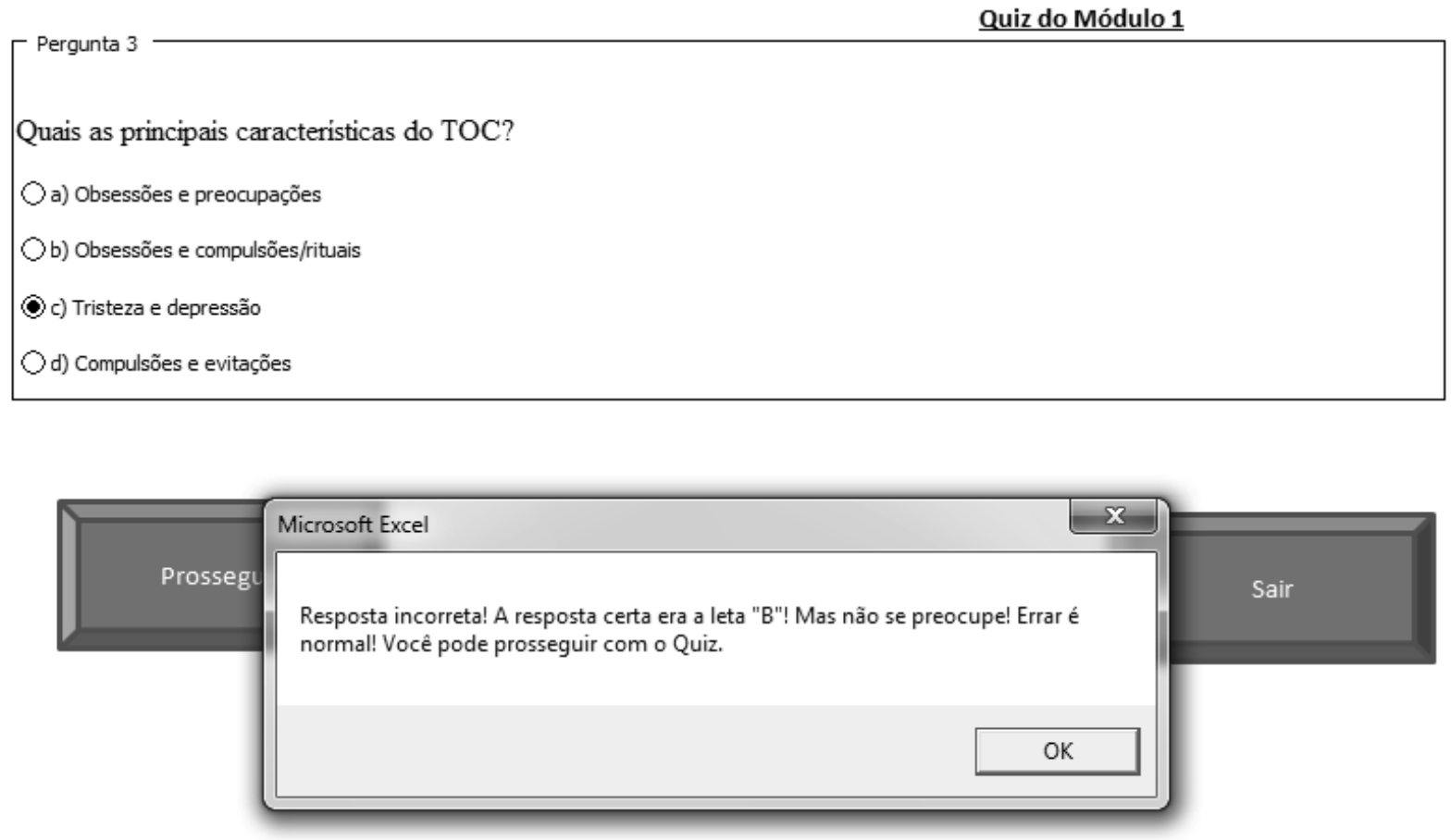

Figura 2. Exemplo de janela do programa (Quiz). 


\section{Considerações Éticas}

A pesquisa foi aprovada pelo Comitê de Ética em Pesquisa da Universidade Federal do Rio Grande do Sul e os participantes assinaram o Termo de Consentimento Livre e Esclarecido. Foram respeitadas as orientações éticas da resolução 466/12 do Conselho Nacional de Saúde e da resolução 011/2012 do Conselho Federal de Psicologia.

\section{Análise dos Dados}

$\mathrm{O}$ teste de Friedman foi utilizado para comparar as médias das escalas de sintomas de TOC, de humor e de ansiedade. Em seguida, utilizou-se o teste de Wilcoxon para verificar onde houve diferença significativa. $\mathrm{Na}$ análise da Y-BOCS, também foi utilizado o teste de Wilcoxon. Os tempos das páginas e acertos nos quizzes são apresentados de forma descritiva, assim como as questões de habilidade com a tecnologia e de usabilidade do programa.

\section{Resultados}

A amostra contou com 21 participantes, entre 19 e 55 anos, com média de idade de 30,29 anos $(D P=10,57)$. Destes, $52,4 \%$ eram homens e $57,1 \%$ possuíam ensino superior incompleto. A amostra foi composta principalmente por moradores de Porto Alegre (66,27\%), com curso superior incompleto $(57,1 \%)$ em diferentes áreas.

Em relação ao uso de computador, $71,4 \%$ dos participantes relataram utilizar computador todos os dias, sendo que $38,1 \%$ somam de 6 a 10 horas de uso por dia. Além disso, em uma escala de 0 a 4 sobre o quanto gostam de usar o computador, 52,4\% responderam " 3 ". O nível de habilidade com o computador mais relatado foi habilidade "média" (52,4\%).

Todos os participantes finalizaram os três módulos. O tempo médio para terminar o programa foi de 7,97 dias $(D P=4,35)$, ou seja, 191,28 horas. O tempo médio passado utilizando o programa foi de 02:14:32 ( $D P=00: 58: 31)$. O tempo médio dos módulos 1,2 e 3 foi de 00:48:32
$(D P=00: 36: 59), 00: 49: 42(D P=00: 37: 47)$ e 00:40:20 ( $D P=00: 21: 07)$, respectivamente.

A média dos tempos por página foi maior nas páginas de preenchimento da Y-BOCS nos dois momentos (10:28'" e 6:03' respectivamente) e na tarefa do módulo 2 (21:41"). "Picos" de menor tempo ocorreram nas páginas de definição das compulsões $(0: 11$ ") e de revisão do humor nos módulos 2 (0:07') e 3 (0:08').

A Tabela 1 apresenta as questões dos quizzes e os respectivos acertos em frequência e percentual. No quiz 1, nenhum participante acertou menos de 6 questões. Quatro participantes escolheram repetir este quiz. A questão 6 foi a que levou mais tempo para ser respondida, com uma média de 55 segundos e a questão 3 teve média de 14 segundos, sendo esta a que levou menos tempo. No segundo quiz, nenhum participante acertou menos de 7 questões. Dois participantes escolheram repetir este quiz. A média de tempo da questão 5 foi de 58 segundos, enquanto a questão 1 levou em média apenas 10 segundos para ser respondida. Por fim, no quiz 3, também nenhum participante acertou menos de 7 questões. Três participantes escolheram repetir este quiz. A questão 1 teve a maior média de tempo de todas as questões do programa: 2 minutos e 19 segundos, enquanto a que levou menos tempo foi a questão 7, com 17 segundos.

O nível médio de satisfação com o primeiro módulo foi de 8,33 ( $D P=1,197)$, sendo 8,71 $(D P=1,056)$ no segundo e $9,00(D P=0,918)$ no terceiro. Apenas um participante relatou satisfação nível 6 (no módulo 1), os demais valores sempre foram 7 ou mais em todos os módulos. Nenhum usuário respondeu "não" para qualquer das cinco perguntas. Conforme pode ser visto na Tabela 2 a frequência das respostas positivas permaneceu sempre acima de 70\%. Das sugestões registradas, $62,5 \%$ indicaram que o programa poderia mudar o aspecto visual para se tornar mais atrativo e $43,7 \%$ recomendaram alterações de funcionalidade, como corrigir falhas nos botões e no feedback automático. 
Tabela 1

Questões, Frequência e Percentual de Acertos nos Quizzes

Questão $f$ Acertos \% Acertos

Quiz 1

O que é o transtorno obsessivo-compulsivo?

Qual a prevalência (percentual estimado) do TOC?

Quais as principais características do TOC?

81

O que são obsessões?

As compulsões são...

O que são as evitações?

Checar repetidamente a porta da geladeira é um exemplo de...

Pensamentos involuntários sobre agredir um amigo é um exemplo de...

Evitar encostar em corrimão de ônibus ou metrô, é um exemplo de...

Tentar substituir um pensamento "ruim" por um pensamento "bom" é um ex de:

Em relação às causas do TOC (qual a causa mais provável?)

Escolha a alternativa verdadeira em relação à neurobiologia do TOC.

Que aspecto cognitivo diferencia o indivíduo com TOC?

Existem medicamentos que melhoram os sintomas do TOC?

O que acontece quando você tem uma recompensa após determinado comportamento?

Geralmente, o que acontece quando você evita fazer algo que causa ansiedade?

Ao evitar uma situação, qual a probabilidade de repetir o comportamento?

Qual a finalidade dos rituais/compulsões?

Ao longo do tempo, o que pode ocorrer com as evitações e rituais/compulsões?

Evitar situações que causam ansiedade resolve o problema?

Quiz 3

Qual o motivo principal do surgimento da TCC na década de 60?

Por que a TCC tem esse nome?

Qual o princípio básico da terapia cognitiva?

Qual o princípio básico da terapia comportamental?

A TCC é eficaz?

Sobre pensamentos e comportamentos, qual alternativa é verdadeira?

Sobre as características da TCC, escolha a alternativa verdadeira...

Sobre as características da TCC, escolha a alternativa verdadeira...

Sobre as características da TCC, escolha a alternativa verdadeira... 
Tabela 2

Frequência e Porcentagem de Respostas para as Questões de Usabilidade

\begin{tabular}{cccc}
\hline Módulos & Item & \% Sim & \% Parcialmente \\
\hline \multirow{3}{*}{ Módulo 1 } & Eficaz/Efetivo & 95,2 & $4,8 \%$ \\
& Eficiente & 85,7 & 14,3 \\
& Compreensível & 90,5 & 9,5 \\
& Confortável & 81,0 & 19,0 \\
& Satisfeito & 71,4 & 28,6 \\
& & & \\
Módulo 2 & Eficaz/Efetivo & 90,5 & 9,5 \\
& Eficiente & 90,5 & 9,5 \\
& Compreensível & 90,5 & 9,5 \\
& Confortável & 85,7 & 14,3 \\
& Satisfeito & 95,2 & 4,8 \\
& & & 5,0 \\
& Eficaz/Efetivo & 95,0 & 0 \\
Módulo 3 & Eficiente & 100 & 15,0 \\
& Compreensível & 85,0 & 5,0 \\
& Confortável & 95,0 & 0 \\
\hline
\end{tabular}

Nas perguntas de triagem, 33,3\% dos participantes relatou ter pensamentos que invadem a mente contra a vontade, $33,3 \%$ comportamentos que não consegue se impedir de fazer e $38,1 \%$ evitação de algum tipo de situação/lugar/pessoas porque lhe causam desconforto, ansiedade ou nojo. Nos resultados da Y-BOCS, a mediana foi de $4(M=4,72 ; D P=4,94)$ no primeiro momento de avaliação e de $2(M=3,84 ; D P=4,89)$ no segundo momento, sendo que os escores variaram entre 0 e 18 e 0 e 16 , respectivamente. O teste de Wilcoxon revelou uma diferença de $1,754(p=0,079)$ na seção de obsessões entre os dois momentos de avaliação. No valor total e no valor das compulsões não houve diferença significativa.

$\mathrm{Na}$ avaliação subjetiva dos sintomas de TOC, as médias de postos nos três tempos foram de 2,52, 1,81 e 1,67 respectivamente. O teste de Friedman indicou um qui-quadrado de 13,527 $(p<0,001)$. Como pode ser visto na Tabela 3 , o teste de Wilcoxon revelou que uma diferença estatisticamente significativa aconteceu entre os tempos 1 e $2(p=0,007)$ e entre os tempos 1 e $3(p=0,002)$. Não houve diferença significativa nos escores de humor e ansiedade.

\section{Discussão}

Este estudo buscou avaliar a usabilidade, funcionalidade e efeitos iniciais de uma intervenção computadorizada através de um ensaio com usuários. O número de usuários recomendado para uma avaliação adequada de usabilidade, em torno de 15, foi alcançada (Kushniruk, Patel, \& Cimino, 1997).

Um imprevisto técnico limitou o tempo mínimo de utilização em seis dias, ao invés de três. Logo, a média de oito dias foi adequada a esta expectativa. Em outras intervenções computadorizadas, a expectativa usualmente é que a pessoa utilize um módulo por semana (Craske et al., 2009; Currie et al., 2010). Além disso, pode haver um terapeuta acompanhando o usuário, o que resulta em um direcionamento maior do tempo (Anderson et al., 2007). Embora o programa fos- 
Tabela 3

Teste de Wilcoxon para os Sintomas de TOC Antes e Depois

\begin{tabular}{|c|c|c|c|c|c|c|}
\hline & \multicolumn{3}{|c|}{ Sintomas Subjetivos } & \multicolumn{3}{|c|}{ Y-BOCS } \\
\hline & \multicolumn{3}{|c|}{ Três tempos } & Total & Obs & Comp \\
\hline & M1 - M2 & M1- M3 & M2 - M3 & M1-M3 & M1 - M3 & M1 -M3 \\
\hline$Z$ & 2,675 & 3,086 & 1,218 & 1,459 & 1,754 & 0,425 \\
\hline$p$ & 0,007 & 0,002 & 0,223 & 0,144 & 0,079 & 0,671 \\
\hline
\end{tabular}

Notas. Y-BOCS = Yale-Brown Obsessive-Compulsive Scale. M1 = Módulo 1, M2 = Módulo 2, M3 = Módulo 3; Obs $=$ Subescala de obsessões; Comp $=$ Subescala de Compulsões

se autoadministrado, é possível que o primeiro e-mail de monitoramento enviado pelo pesquisador possa ter influenciado no tempo de uso, uma vez que constitui intervenção humana, ainda que não seja terapêutica (Newman, Szkodny, Llera, \& Przeworski, 2011).

Os tempos de uso relativamente curtos são um aspecto importante, já que programas longos demais podem ser cansativos e desagradáveis Anderson e colaboradores (2007) relataram tempos semelhantes em sua intervenção computadorizada para ansiedade social: os tempos variaram entre 5 e 45 minutos por módulo, com média de 30 minutos. O tempo curto de uma sessão computadorizada pode ser um bom indicador de usabilidade (Anderson et al., 2007). Casos em que o tempo de uso foi muito curto podem indicar que o usuário já tinha conhecimento prévio do assunto. Por outro lado, onde uma quantidade maior de tempo foi utilizado, é possível que essas pessoas estivessem envolvidas em outras atividades quando da utilização do programa. Essa falta de dados sobre o ambiente do paciente é uma característica das intervenções online e pode ser encontrada também por psicólogos que oferecem orientação psicológica online em sua prática profissional (Siegmund \& Lisboa, 2015). Com relação ao tempo médio utilizado em cada página, em geral foram proporcionais com a quantidade de informação presente na tela ou nível de atenção necessário para realizar o que era solicitado.

Em relação aos quizzes, altas frequências de acertos significam que o paciente ou usuário assimilou a lógica do tratamento, o que por sua vez é preditor de melhores resultados (Abramo- witz et al., 2002). Essa é uma condição favorável, pois à medida que um paciente compreende melhor o transtorno e o tratamento, ele tem mais condições de lidar com seus sintomas e de aderir à terapia. Essa mesma técnica foi utilizada em um estudo com usuários de maconha e a assimilação do conteúdo influenciou positivamente os resultados do tratamento (Budney et al., 2011). $\mathrm{O}$ menor número de acertos em alguns quizzes pode indicar uma necessidade de reformular o texto da respectiva questão, ou mesmo uma alteração na formulação da pergunta. Existe também a possibilidade de o tema não despertar interesse para alguém que não apresenta sintomas de TOC. Uma característica secundária dos quizzes é que eles trazem uma dinâmica agradável ao programa.

O nível de satisfação com o programa foi considerado adequado, ficando com média acima de 8 para todos os módulos. Com base em resultados negativos de outros estudos (Christensen, Griffiths, Mackinnon, \& Brittliffe, 2006; Ellis, Campbell, Sethi, \& O'Dea, 2011), é possível considerar que o programa tem um bom potencial de aceitação e que não são necessárias alterações maiores. O único critério da usabilidade que ficou abaixo de $80 \%$ de aceitação dos usuários foi a satisfação com o módulo 1 , cuja explicação pode ser a falta de familiaridade inicial com o software ou a falta de atratividade visual relatada por grande parte dos usuários.

Encontramos uma diferença tendendo à significância estatística na subescala de obsessões, sugerindo que os sintomas obsessivos reduziram até o final da intervenção. É importante ressal- 
tar que essa diferença ocorreu com o uso de um programa sem acompanhamento de terapeuta e no intervalo médio de apenas oito dias. Tem-se sugerido que intervenções sem acompanhamento mínimo de um terapeuta tendem a ser menos efetivas (Newman et al., 2011). Em geral os tratamentos tradicionais para TOC tem duração mínima de quatro semanas quando são intensivas (Foa et al., 2005) e não encontramos estudos que tenham avaliado a influência isolada da psicoeducação na redução de sintomas do TOC. Deve-se considerar que os usuários possam simplesmente ter aprendido a avaliar melhor seus sintomas. Este também seria um avanço importante em termos de saúde pública, pois um dos objetivos das intervenções autoadministradas é justamente empoderar os pacientes, torná-los mais autônomos, conhecedores da sua condição e responsáveis pelo seu tratamento (Cartreine, Ahern, \& Locke, 2010).

As pontuações na escala Y-BOCS, com média e mediana abaixo de sete,indicam que a amostra situa-se na faixa de sintomas subclínicos segundo a classificação do instrumento. Contudo, ressalta-se que grupos não clínicos também podem se beneficiar da psicoeducação como um processo componente de alfabetização psicológica. Ademais, uma diferença estatisticamente significativa dos sintomas na avaliação subjetiva de TOC também foi encontrada, indicando redução nos sintomas percebidos. O Y-BOCS no formato informatizado tem potencial para ser adequadamente administrado via computador e ajuda a minimizar o tempo utilizado pelo terapeuta nos tratamentos.

O estudo aqui apresentado tem limitações quanto à implementação, teste e avaliação que merecem ser ressaltadas, informando futuras iniciativas semelhantes de desenvolvimento de programas de intervenção online. A primeira limitação é a ausência de uma avaliação qualitativa com os usuários ao longo e ao final do programa (Kushniruk \& Patel, 2004). Um segundo limitador é a impossibilidade de controle sobre outras atividades concomitantes com a utilização do software. Outrossim, cumpre ressaltar que, nesse formato, o estudo apresentou uma si- tuação muito próxima da realidade dos futuros usuários. Um terceiro limitador sugere uma avaliação diagnóstica mais criteriosa, para identificar outros transtornos e discriminar os diferentes sintomas de TOC. Finalmente, por se tratar de um estágio de desenvolvimento do software, não foi possível selecionar uma amostra clínica para testar a eficácia, o que deverá ser realizado posteriormente.

Apesar dessas limitações, entendemos que a intervenção foi avaliada de forma positiva pelos usuários e representa uma contribuição teórico-prática importante para o campo. Uma das principais expectativas é transformar o programa em um aplicativo que possa ser utilizado em ipads, iphones e tablets, como já foi tentado com o transtorno do pânico e a ansiedade social (e.g., Lindner, Ivanova, Ly, Andersson, \& Carlbring, 2013). Atualmente, cerca de $15 \%$ da população utiliza Internet móvel e considerando o cenário atual, em breve o Brasil será "mobile" (Instituto Brasileiro de Opinião Pública e Estatística [IBOPE] Media, 2013). Para as intervenções em psicologia, esse formato facilita a utilização, potencializa o uso de tempo e permite ter acesso às informações do usuário em tempo real.

\section{Conclusão}

A introdução de uma intervenção psicoeducacional computadorizada, especialmente no Brasil, pode trazer benefícios para profissionais e usuários. Intervenções computadorizadas seriam facilmente disseminadas na rede pública, a exemplo da Inglaterra (Powell et al., 2013), aumentando o conhecimento da população sobre saúde mental, psicopatologia e tratamentos disponíveis. Por ser uma intervenção psicoeducacional, qualquer pessoa interessada pode utilizá-la. Portanto, esse tipo de intervenção poderia atuar na prevenção em saúde mental e identificação precoce de psicopatologias, que é um aspecto fundamental e desejável em nosso país (Cordeiro, Oliveira, Melzer, Ribeiro, \& Rigonatti, 2010).

Uma outra possibilidade é que essas intervenções sejam usadas em conjunto com o tra- 
tamento psicológico tradicional, melhorando o engajamento dos pacientes, facilitando a realização de tarefas de casa e potencializando os impactos da terapia (Budney et al., 2011; Carroll et al., 2008; Lind et al., 2013). É sugerido que intervenções computadorizadas podem ser aplicadas de forma autoadministrada como uma primeira fase do tratamento psicológico, onde a segunda etapa seria uma intervenção com acompanhamento à distância de um técnico ou clínico e a terceira fase o tratamento presencial (Titov et al., 2010).

Existem inúmeras possibilidades para estudos futuros com o próprio programa aqui desenvolvido. O primeiro deles seria avaliar a efetividade do programa para pessoas com sintomas clínicos de TOC. Em seguida, torna-se fundamental comparar uma intervenção de psicoeducação com uma intervenção tradicional e observar as diferenças de efetividade, adesão e experiência. Por fim, consideramos que seria de grande importância para o campo comparar intervenções em que há um terapeuta virtual participativo e intervenções onde não há um terapeuta virtual.

\section{Referências}

Abramowitz, J. S., Franklin, M. E., Zoellner, L. A., \& DiBernardo, C. L. (2002). Treatment compliance and outcome in obsessive-compulsive disorder. Behavior Modification, 26(4), 447-463.

American Psychiatric Association. (2013). Diagnostic and statistical manual of mental disorders: DSM-5. Washington, DC: Author.

Anderson, P., Zimand, E., Schmertz, S. K., \& Ferrer, M. (2007). Usability and utility of a computerized cognitive-behavioral self-help program for public speaking anxiety. Cognitive and Behavioral Practice, 14(2), 198-207. doi:10.1016/j. cbpra.2006.02.006

Andersson, E., Enander, J., Andrén, P., Hedman, E., Ljótsson, B., Hursti, T., ...Rück, C. (2012). Internet-based cognitive behaviour therapy for obsessive-compulsive disorder: A randomized controlled trial. Psychological Medicine, 42(10), 2193-2203. doi:10.1017/S0033291712000244

Andersson, E., Ljótsson, B., Hedman, E., Kaldo, V., Paxling, B., Andersson, G., ...Rück, C. (2011).
Internet-based cognitive behavior therapy for obsessive compulsive disorder: A pilot study. Boston Medical Center Psychiatry, 11, 125. doi:10.1186/1471-244X-11-125

Andersson, G. (2009). Using the Internet to provide cognitive behaviour therapy. Behaviour Research and Therapy, 47(3), 175-180. doi:10.1016/j.brat.2009.01.010

Asbahr, F., Lotufo, F., Neto, Turecki, G., Del Porto, J., Rodriguez, L., Baruzzi, M., ...Gentil, V. (1992). Escala Yale-Brown de Sintomas Obsessivo-Compulsivos: Tradução brasileira. São Paulo, SP.

Barlow, J. H., Ellard, D. R., Hainsworth, J. M., Jones, F. R., \& Fisher, A. (2005). A review of selfmanagement interventions for panic disorders, phobias and obsessive-compulsive disorders. Acta Psychiatrica Scandinavica, 111(4), 272285. doi:10.1111/j.1600-0447.2005.00499.x

Beck, J. (2007). Terapia cognitiva: Teoria e prática. Porto Alegre, RS: Artmed.

Budney, A. J., Fearer, S., Walker, D. D., Stanger, C., Thostenson, J., Grabinski, M., \& Bickel, W. K. (2011). An initial trial of a computerized behavioral intervention for cannabis use disorder. Drug and Alcohol Dependence, 115(1-2), 74-79. doi:10.1016/j.drugalcdep.2010.10.014

Carroll, K. M., Ball, S. A., Martino, S., Nich, C., Babuscio, T. A., Nuro, K. F., ...Rounsaville, B. J. (2008). Computer-assisted delivery of cognitivebehavioral therapy for addiction: A randomized trial of CBT4CBT. The American Journal of Psychiatry, 165(7), 881-888. doi:10.1176/appi. ajp.2008.07111835

Cartreine, J. A., Ahern, D. K., \& Locke, S. E. (2010). A roadmap to computer-based psychotherapy in the United States. Harvard Review of Psychiatry, 18(2), 80-95. doi:10.3109/10673221003707702

Castelnuovo, G., Gaggioli, A., Mantovani, F., \& Riva, G. (2003). From psychotherapy to e-therapy: The integration of traditional techniques and new communication tools in clinical settings. Cyberpsychology \& Behavior, 6(4), 375-382. doi:10.1089/109493103322278754

Christensen, H., Griffiths, K. M., Mackinnon, A. J., \& Brittliffe, K. (2006). Online randomized controlled trial of brief and full cognitive behaviour therapy for depression. Psychological Medicine, 36(12), 1737-1746. doi:10.1017/ S0033291706008695 
Colom, F. (2014). The evolution of psychoeducation for bipolar disorder: From lithium clinics to integrative psychoeducation. World Psychiatry, 13(1), 90-92. doi:10.1002/wps.20091

Conselho Federal de Psicologia. (2012, 12 jun.). Resolução $C F P N^{\circ}$ 011/ 2012. Recuperado em http:// site.cfp.org.br/wp-content/uploads/2012/07/Resoluxo_CFP_nx_011-12.pdf

Cordeiro, Q., Oliveira, A. M. de, Melzer, D., Ribeiro, R. B., \& Rigonatti, S. P. (2010). Prevenção em saúde mental. Revista do Curso de Direito, 7(7), 38-53.

Corrao, N. J., Robinson, A. G., Swiernik, M. A., \& Naeim, A. (2010). Importance of testing for usability when selecting and implementing an electronic health or medical record system. Journal of Oncology Practice, 6(3), 120-124. doi:10.1200/JOP.200017

Craske, M. G., Rose, R. D., Lang, A., Welch, S. S., Campbell-Sills, L., Sullivan, G., ...Roy-Byrne, P. P. (2009). Computer-assisted delivery of cognitive behavioral therapy for anxiety disorders in primary-care settings. Depression and Anxiety, 26(3), 235-242. doi:10.1002/da.20542

Crino, R., Slade, T., \& Andrews, G. (2005). The changing prevalence and severity of obsessivecompulsive disorder criteria from DSM-III to DSM-IV. The American Journal of Psychiatry, 162(5), 876-882. doi:10.1176/appi. ajp.162.5.876

Currie, S. L., McGrath, P. J., \& Day, V. (2010). Development and usability of an online CBT program for symptoms of moderate depression, anxiety, and stress in post-secondary students. Computers in Human Behavior, 26(6), 14191426. doi:10.1016/j.chb.2010.04.020

Dowrick, C., Dunn, G., Ayuso-Mateos, J. L., Dalgard, O. S., Page, H., Lehtinen, V., ...Wilkinson, G. (2000). Problem solving treatment and group psychoeducation for depression: Multicentre randomised controlled trial. British Medical Journal, 321(7274), 1450. doi:10.1136/ bmj.321.7274.1450

Ellis, L. A., Campbell, A. J., Sethi, S., \& O’Dea, B. M. (2011). Comparative randomized trial of an online cognitive-behavioral therapy program and an online support group for depression and anxiety. Journal of CyberTherapy and Rehabilitation, 4(4), 461-467.
Foa, E. B., Liebowitz, M. R., Kozak, M. J., Davies, S., Campeas, R., Franklin, M. E., ...Tu, X. (2005). Randomized, placebo-controlled trial of exposure and ritual prevention, clomipramine, and their combination in the treatment of obsessivecompulsive disorder. The American Journal of Psychiatry, 162(1), 151-161. doi:10.1176/appi. ajp.162.1.151

Franklin, M. E., Abramowitz, J. S., Bux, D. A., Jr., Zoellner, L. A., \& Feeny, N. C. (2002). Cognitive-behavioral therapy with and without medication in the treatment of obsessive-compulsive disorder. Professional Psychology: Research and Practice, 33(2), 162-168. doi:10.1037/07357028.33.2.162

Goodman, W. K., Price, L. H., Rasmussen, S. A., Mazure, C., Fleischmann, R. L., Hill, C. L., ... Charney, D. S. (1989). The Yale-Brown Obsessive Compulsive Scale. I. Development, use, and reliability. Archives of General Psychiatry, 46(11), 1006-1011.

Goodwin, R., Koenen, K. C., Hellman, F., Guardino, M., \& Struening, E. (2002). Helpseeking and access to mental health treatment for obsessive-compulsive disorder. Acta Psychiatrica Scandinavica, 106(2), 143-149.

Herbst, N., Voderholzer, U., Stelzer, N., Knaevelsrud, C., Hertenstein, E., Schlegl, S., ...Külz, A. K. (2012). The potential of telemental health applications for obsessive-compulsive disorder. Clinical Psychology Review, 32(6), 454-466. doi:10.1016/j.cpr.2012.04.005

Houghton, S., \& Saxon, D. (2007). An evaluation of large group CBT psycho-education for anxiety disorders delivered in routine practice. Patient Education and Counseling, 68(1), 107-110. doi:10.1016/j.pec.2007.05.010

Instituto Brasileiro de Opinião Pública e Estatística Media. (2013, 15 jul.). Internet móvel avança no Brasil. Recuperado em http://www.ibope.com. br/pt-br/conhecimento/artigospapers/Paginas/ Internet-movel-avan\%C3\%A7a-no-Brasil.aspx

International Organization for Standardization 924111. (1998). Ergonomic requirements for office work with visual display terminals (VDTs) -Part 11: Guidance on usability.

Kim, C.-J., \& Kang, S. (2013). Development and a pilot test of an internet-based cardiovascular risk reduction program for Korean male workers 
with metabolic syndrome. CIN: Computers, Informatics, Nursing, 31(4), 157-166. doi:10.1097/ NXN.0b013e3182812829

Klein, B., Mitchell, J., Abbott, J., Shandley, K., Austin, D., Gilson, K., ...Redman, T. (2010). A therapist-assisted cognitive behavior therapy internet intervention for posttraumatic stress disorder: Pre-, post- and 3-month follow-up results from an open trial. Journal of Anxiety Disorders, 24(6), 635-644. doi:10.1016/j.janxdis.2010.04.005

Koran, L. M., Hanna, G. L., Hollander, E., Nestadt, G., Simpson, H. B., \& American Psychiatric Association. (2007). Practice guideline for the treatment of patients with obsessive-compulsive disorder. The American Journal of Psychiatry, 164(Suppl. 7), 5-53.

Kushniruk, A. W., \& Patel, V. L. (2004). Cognitive and usability engineering methods for the evaluation of clinical information systems. Journal of Biomedical Informatics, 37(1), 56-76. doi:10.1016/j.jbi.2004.01.003

Kushniruk, A. W., Patel, V. L., \& Cimino, J. J. (1997). Usability testing in medical informatics: Cognitive approaches to evaluation of information systems and user interfaces. Proceedings of the AMIA Annual Fall Symposium, 218-222.

Lehman, A. F., Lieberman, J. A., Dixon, L. B., McGlashan, T. H., Miller, A. L., Perkins, D. O., ...Steering Committee on Practice Guidelines. (2004). Practice guideline for the treatment of patients with schizophrenia, second edition. The American Journal of Psychiatry, 161(Suppl. 2), $1-56$.

Lind, C., Boschen, M. J., \& Morrissey, S. (2013). Technological advances in psychotherapy: Implications for the assessment and treatment of obsessive compulsive disorder. Journal of Anxiety Disorders, 27(1), 47-55. doi:10.1016/j.janxdis.2012.09.004

Lindner, P., Ivanova, E., Ly, K. H., Andersson, G., \& Carlbring, P. (2013). Guided and unguided CBT for social anxiety disorder and/or panic disorder via the Internet and a smartphone application: Study protocol for a randomised controlled trial. Trials, 14(1), 437. doi:10.1186/1745-6215-14437

Newman, M. G., Szkodny, L. E., Llera, S. J., \& Przeworski, A. (2011). A review of technologyassisted self-help and minimal contact the- rapies for anxiety and depression: Is human contact necessary for therapeutic efficacy? Clinical Psychology Review, 31(1), 89-103. doi:10.1016/j.cpr.2010.09.008

Patterson, E. S., Zhang, J., Abbott, P., Gibbons, M. C., Lowry, S. Z., Quinn, M. T., ...Brick, D. (2013). Enhancing electronic health record usability in pediatric patient care: A scenario-based approach. Joint Commission Journal on Quality and Patient Safety, 39(3), 129-135.

Pier, C., Austin, D. W., Klein, B., Mitchell, J., Schattner, P., Ciechomski, L., ...Wade, V. (2008). A controlled trial of internet-based cognitive-behavioural therapy for panic disorder with face-to-face support from a general practitioner or email support from a psychologist. Mental Health in Family Medicine, 5(1), 29-39.

Powell, J., Hamborg, T., Stallard, N., Burls, A., McSorley, J., Bennett, K., ...Christensen, H. (2013). Effectiveness of a web-based cognitivebehavioral tool to improve mental well-being in the general population: Randomized controlled trial. Journal of Medical Internet Research, 15(1), e2. doi:10.2196/jmir.2240

Preece, J., Carey, T., Rogers, Y., Holland, S., Sharp, H., \& Benyon, D. (1994). Human-computer interaction. Reading, MA: Addison-Wesley.

Rachman, S. (2006). Fear of contamination: Assessment and treatment. Oxford, UK: Oxford University Press.

Rhebergen, M. D. F., Hulshof, C. T. J., Lenderink, A. F., \& van Dijk, F. J. H. (2010). An online network tool for quality information to answer questions about occupational safety and health: Usability and applicability. Boston Medical Center Medical Informatics and Decision Making, 10, 63. doi:10.1186/1472-6947-10-63

Riiser, K., Løndal, K., Ommundsen, Y., Sundar, T., \& Helseth, S. (2013). Development and usability testing of an Internet intervention to increase physical activity in overweight adolescents. Journal of Medical Internet Research Research Protocols, 2(1), e7. doi:10.2196/resprot.2410

Shapiro, D. A., Cavanagh, K., \& Lomas, H. (2003). Geographic inequity in the availability of cognitive behavioural therapy in England and Wales. Behavioural and Cognitive Psychotherapy, 31(2), 185-192. doi:10.1017/ S1352465803002066 
Siegmund, G., \& Lisboa, C. S. de M. (2015). Orientação psicológica online: Percepção dos profissionais sobre a relação com os clientes. Psicologia: Ciência e Profissão, 35(1), 168-181. doi:10.1590/1982-3703001312012

Titov, N., Andrews, G., \& Sachdev, P. (2010). Computer-delivered cognitive behavioural therapy: Effective and getting ready for dissemination. Faculty of 1000 Medicine Reports, 2. doi:10.3410/M2-49

Vernmark, K., Lenndin, J., Bjärehed, J., Carlsson, M., Karlsson, J., Oberg, J., ...Andersson, G. (2010). Internet administered guided self-help versus individualized e-mail therapy: A randomized trial of two versions of CBT for major depression. Behaviour Research and Therapy, 48(5), 368376. doi:10.1016/j.brat.2010.01.005
Wootton, B. M., Titov, N., Dear, B. F., Spence, J., Andrews, G., Johnston, L., \& Solley, K. (2011). An Internet administered treatment program for obsessive-compulsive disorder: A feasibility study. Journal of Anxiety Disorders, 25(8), 1102-1107. doi:10.1016/j.janxdis.2011.07.009

Recebido: 17/09/2014

$1^{a}$ revisão: 06/03/2015

Aceite final: 10/03/2015 